\title{
LE DESTIN DE L'ART GREC
}

En Occident, l'art grec continue de poser de difficiles problèmes aux philosophes. Cette difficulté s'accentue avec le développement tumultueux et l'extrême diversification de l'art contemporain. Comment concilier l'admiration pour l'Aphrodite de Praxitèle et pour une baigneuse "éclatée" de Picasso, pour le Panthéon d'Athènes et pour le Centre Pompidou à Paris?

Certes, beaucoup de nos contemporains, visitant les Antiques du Louvre, s'abandonnent sans autre inquiétude à la contemplation heureuse des statues. Ils ne se posent pas de problemes théoriques a propos du sentiment qu'ils éprouvent et des jugements implicites qu'ils portent. Mais quelques esprits plus exigeants s'interrogent sur la nature de leur rapport à l'art grec, et ceci d'autant plus vivement que se répand en cette partie du monde ce que l'on peut appeler une "idéologie de la rupture": selon la perspective générale qu'elle ouvre, il n'y aurait au fond, et malgré quelques apparences, aucune relation de filiation, de causalité, et donc aucune continuité véritable, entre les connaissance scientifiques passées et la science de notre temps, entre les conceptions philosophiques d'autrefois et les nôtres. Alors pourquoi une coupure aussi profonde, ou même absolue, ne s'établirait-elle pas entre l'art ancien et l'art actuel? Des mutations radicales se produiraient dans ce domaine comme dans les autres.

Même si l'on n'adopte pas des vues aussi extrêmes, la différence profonde de l'art antique ne manque pas de provoquer quelque embarras. Il semble qu'un philosophe l'a ressenti plus fortement que beaucoup d'autres, et de manière en quelque sorte exemplaire: Marx. Il a laissé de précieuses réflexions à ce propos.

On comprend bien sa sensibilité particulière. Il avait élaboré une théorie matérialiste globale du développement historique, dans laquelle il montrait que les "superstructures" et la vie spirituelle -- par exemple la politique, le droit. l'idéologie -- dependent des modifications successives de la base matérielle des sociétés humaines. Chacune de ces instances apparaît ou depérit, progresse au même rythme que la base, en dernière instance. Dans de célebres pages de l'Introduction à la critique de l'economic politique, il se pose ouvertement la question: ne doit-il pas en être de même de l'art? Et il repond d'une manière qui peut déconcerter d'abord le lecteur: "Certaines époques de floraison 
artistique ne sont nullement en rapport avec le développement général de la société". ${ }^{1} \mathrm{Et}$, bien sûr, il propose immédiatement comme exemple l'art grec antique.

L'apparition historique d'un nouveau type de vie économique et sociale n'impliquait donc pas à ses yeux la peremption des formes artistiques issues des types anciens. Il ne conseillait certes pas la destruction des oeuvres d'art du passé, ni la négligence à leur égard! Il savait pourtant que souvent, en d'autres époques, un nouveau cadre social, une nouvelle idéologie, une nouvelle religion avaient pu provoquer un tel vandalisme. Et ce vandalisme fut parfois glorifie par les époques ultérieures. Ainsi dans une de ses tragédies, Polyeucte, Corneille fait-il l'apologie du geste d'un prosélyte chrétien brisant les statues des dieux anciens. Une "païenne" raconte, dans la pièce, ce sacrilege odieux à ses yeux:

Du plus puissant des dieux nous voyons la statue

Par une main impie à leurs pieds abattue,

Les mystères troublés, les temples profanés"... mêmes!

C'est que les belles statues étaient pour les Grecs les dieux eux-

Mais du simple point de vue esthétique, le sacrilège se renouvelle quotidiennement de nos jours. Par exemple, récemment, dans une ville française, les bulldozers, pour creuser un parking automobile souterrain, ont saccagé promptement et subrepticement, afin d'éviter toute recrimination importune, les restes enfouis depuis longtemps d'une basilique byzantine, avec ses mosaïques remarquables. Hegel avait justement posé la question: "Pourquoi notre époque n'est-elle pas favorable à l'art, en général?". Et Marx avait aiguisé cette question: "Pourquoi notre époque est-elle hostile à l'art et à la poésie?"2 $\mathrm{La}$ réponse était évidente: ce sont les intérêts mercantiles, le pouvoir de l'argent, le capitalisme, qui entravent le développement de l'art.

L'attitude de Marx à l'egard de l'art, en son temps, ne peut se comprendre si l'on ne prend en compte son respect infini pour l'art grec, son admiration véritablement enthousiaste pour lui. Cela exige un effort de notre part car, sans bien sûr renier l'art grec, nous ne lui accordons sans doute plus la même exclusivité, à cause de l'ampleur, en quantité, et de la richesse, en variété extrême, des productions de l'art pendant le dernier siècle.

${ }^{1}$ Marx. Contribution à la Critique de l'economie politique. Introdution, Trad. par Husson et Badia. Paris, 1957, p. 173.

${ }^{2}$ Marx-Engels-Werks, tome XXVI (I). Berlin: 1965, p. 257. 
Mais à l'époque de Marx, et dans les temps qui la précèdent immédiatement, l'art grec domine hégémoniquement les esprits. Cela ne témoigne pas d'un état d'esprit retardataire. Au contraire, ce sont les esprits les plus éminents et les plus progressistes qui témoignent alors de cette preférence. Marx, qui critique radicalement Hegel en certains domaines, partage sur ce point son jugement. Hegel explique longuement, et à plusieurs reprises, qu'en Grèce "l'art a atteint le sommet"3. Mais, en cela, il reprend les thèses que Georges Forster, le révolutionnaire que l'on a surnommé "le Jacobin allemand", avait lui-même développées: "les Grecs se sont élevés tout seuis jusqu'à la plus haute perfection de l'Idéal"4. Certes d'autres Allemands soutenaient la même opinion, mais Hegel avait lu Forster d'une maniere particulièrement attentive. C'est donc dans cette lignee progressiste que Marx se range lorsqu'il écrit que les chefs d'oeuvre de l'art grec "ont encore pour nous, à certains égards, la valeur de normes et de modèles inaccessibles" 5 . Voilà ce que l'art grec propose aux artistes du XIXè siècle: des "modèles inaccessibles", à certains égards!

Cet engouement pour la Grèce s'exagère peut-être excessivement. Il s'accroissait d'autant plus que Hegel et Forster se sentaient plus déçus par le monde dans lequel ils vivaient: un monde déchiré, "aliéné", conflictuel, un monde d'exploitation et d'oppression. Par contraste, ils cédaient à la tendance d'idéaliser, d'embellir imaginairement le souvenir de la Grèce antique dont ils s'enchantaient.

On sait que Sophocle est un des auteurs les plus cités par Marx, et jusque dans Le Capital. Quand on lui demandait d'indiquer ses poètes preférés, il répondait: "Shakespeare, Eschyle, Goethe". Son gendre, Paul Lafargue, atteste que Marx "relisait tous les ans Eschyle dans le texte

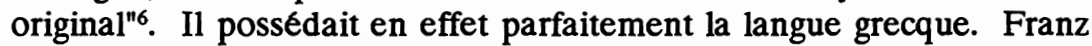
Mehring en témoigne: "Il est resté fidèle à ses vieux Grecs, et tous les misérables épiciers qui voudraient dégoûter les ouvriers de la culture antique, il les aurait chassés du temple avec des verges"7.

Ainsi Mehring en appelle-t-il à une image de la mythologie chrétienne (les marchands du temple) pour protéger, avec Marx, la mémoire de la mythologie païenne grecque!

${ }^{3}$ Hegel, Esthétique. trad. S. Jankélévitch. Paris: 1944, Il, p. 154.

${ }^{4}$ Forster, Georg. Philosophische Schriften. Berlin: Ak. Verlag, 1958, p. 115.

${ }^{5}$ Marx. Contribution... Op. cit., p. 175.

${ }^{6}$ Paul Lafargue: Karl Marx, souvenirs personnels. Die Neue Zeit, 1891.

${ }^{7}$ Mehring, Karl Marx. Leipzig, 1923, pp. 509-510. 
En somme, pour Marx, la difficulté n'est pas "de comprendre que l'art grec et l'épopée sont liés à certaines formes du développement social." Il se fait fort de l'expliquer. Mais il ne dissimule pas le véritable enjeu dans l'horizon théorique qu'il a lui-même ouvert: "La difficulté reside dans le fait qu'ils nous procurent encore une jouissance esthétique"...8.

Concernant cette question, Marx se place délibérément à un point de vue historique. L'héritage hégélien apparaît ici visiblement. Hegel n'a pas su découvrir les fondements d'une véritable histoire de l'art, mais il a affirmé qu'il y a de tels fondements. Il les conçoit de manière idéaliste: chaque période sociale présente une unité de ses diverses instances (politique, religieuse, philosophique, artistique, etc.) qui repose sur ce qu'il appelle lui-même une base, une base spirituelle.

Marx critiquera, évidemment, ce point de vue spiritualiste, mais il s'inspirera de la perspective historique: Il la parcourra parfois jusque dans des détails.

Chez Hegel, il trouvera une étonnante assimilation de l'art grec à la religion: La religion grecque se réduit en quelque sorte à l'art luimême. Cela implique une conception générale de la religion assez hétérodoxe: la religion de l'art, la Kunstreligion.

Nos trois philosophes, Forster, Hegel et Marx s'accordent pour constater le lien étroit de ce qu'ils tiennent pour le plus beau de tous les arts avec la religion du peuple où il est né et s'est épanoui.

En affirmant que "les artistes grecs ont puisé leurs sujets dans la religion nationale (Volksreligion)" 9 , Hegel ratifie ce jugement de Forster: "Ce sont les objets de la religion nationale (Volksreligion) qui, depuis toujours, enflammerent le génie de l'artiste"10. Et d'ailleurs tous deux en appellent sur ce point à l'exemple de Phidias ${ }^{11}$.

Forster l'avait précisé: l'art grec n'a pu naître que grâce à "une heureuse concordance de l'idée de l'art et du système religieux de ces peuples"12. Hegel, de la même manière, reconnaîtra "l'adéquation, la correspondance incluse dans le concept de l'art grec et dans celui de la mythologie grecque ${ }^{13}$.

Marx confirme cette opinion, comme si elle allait de soi, comme

\footnotetext{
${ }^{8}$ Marx. Contribution... Op. cit., p. 175.

${ }^{9}$ Esthetique, II, p. 156.

${ }^{10}$ Forster, Werke (1843), t.V. p. 307.

${ }^{11}$ Forster, Ibid., p. 308; Hegel, Ibid., p. 156.

${ }^{12}$ Forster, Ansichten... (Berlin, 1791), I, p. 204.

${ }^{13}$ Esthétique, II, p. 154-155.
} 
quelque chose de bien connu - mais ce n'est bien connu que depuis Hegel: "On sait que la mythologie grecque n'a pas été seulement l'arsenal de l'art grec, mais la terre même qui l'a nourri (...) l'art grec suppose la mythologie grecque... (...). Jamais la mythologie égyptienne n'aurait pu fournir un terrain favorable à l'éclosion de l'art grec ${ }^{\text {"14 }}$.

Marx ne cautionne pas, du moins expressément, la doctrine hégélienne de la "religion de l'art." Celle-ci, cependant, était beaucoup moins "religieuse" que son nom pourrait le faire croire d'abord. Hegel la conçoit essentiellement comme une sorte de religion de l'homme.

L'idée d'une "religion de l'art" se faisait jour déjà chez Forster. Les oeuvres d'art et la religion grecques sont de la même famille. Grâce à cette parenté, dit Forster, "on éleva ces modèles de beauté et de perfection surhumaines en objets d'adoration ${ }^{n 15}$; grâce à elle, dit Hegel, "l'Art put devenir en Grèce la plus haute expression de l'Absolu, et la religion grecque celle de l'art même" ${ }^{16}$.

Cela ne signifie pas du tout, ni pour Forster, ni pour Hegel, que l'art grec dérive de la religion grecque. Bien au contraire! Les artistes grecs n'ont pas trouvé les dieux déjà existants, ils les ont crées.

Forster décrit longuementl'effort grâce auquel l'artiste forma "la première statue d'un dieu avec les traits de l'humanité transfiguree (verklärt) $)^{\text {n17 }}$.

Ce n'est pas l'homme qui est "crée à l'image de Dieu", comme l'enseignera la Révélation chrétienne, mais c'est le Dieu qui est créé à l'image de l'homme!

Hegel, à propos du rôle de la figure humaine dans l'art, montrera comment "ce moment naturel se trouve completement tranfigure (verklärt) en belle figure". ${ }^{18}$

Il revient sans cesse sur le fait que la mythologie est l'oeuvre des artistes. "Les poètes, dit-il, sont devenus les véritables créateurs de la mythologie que nous admirons dans l'art grec"19.

C'est donc directement à Forster que Hegel a pu emprunter cette idée que "l'artiste est le maître du Dieu"20. Il la développe

\footnotetext{
${ }^{14}$ Marx, op.cit., p. 174.

${ }^{15}$ Forster, Ansichten..., I, 204.

${ }^{16}$ Esthetique, II, p. 154-155.

${ }^{17}$ Forster, Philos. Schriften, p. 126.

${ }^{18}$ Philosophie der Religion (Glockner),XVI, p. 120.

${ }^{19}$ Esthetique, II, p.154-155.

${ }^{20}$ Encyclopedie, paragraphe 560.
} 
longuement dans l'Esthétique. A un stade inférieur du développement de l'esprit, un peuple ne saisit pas la vérité sous une forme conceptuelle, ni ne la développe en un système scientifique, mais il s'en donne la représentation dans des images que l'art objective:

Chez les Grecs, dit Hegel, l'art était la forme la plus haute sous laquelle le peuple se représentait les dieux et prenait conscience de la verité. C'est pourquoi les artistes et les poètes de la Grece étaient devenus les créateurs de ses dieux, c'est-à-dire que les artistes ont donné à leur nation une représentation définie de la vie et des actes des dieux, et à la religion un contenu défini' ${ }^{21}$.

L'art grec n'est l'illustration ni l'auxiliaire d'une religion préexistante. Il exprime directement la vérité telle que la voit le peuple, aussi se propose-t-il comme religion de ce peuple.

Pour Hegel, la beauté la plus haute, c'est l'homme, parce que l'homme est un être moralement social (sittlich). Le sens hégélien de la Sittlichkeit trouve certainement un encouragement chez Forster qui écrit, par exemple:

Je sais que l'on peut véritablement idéaliser l'homme mieux que tous les objets de la nature, car l'Idéal que l'artiste esquisse doit représenter, en même temps que les justes proportions du corps humain en tant qu'espèce animale particulière, aussi la Sittlichkeit, la socialité de l'homme, comme quelque chose de ressenti sympathiquement" 22 .

Hegel expliquera, de son côté, que "l'humain constitue le centre et le contenu de la beauté et de l'art véritable" ${ }^{23}$, car "cette forme humaine est seule capable de révéler le spirituel d'une façon sensible ${ }^{\mathrm{n} 24}$ et l'on rencontrera chez Hegel comme chez Forster l'exaltation de l'nindividualité plastique"25, de "la belle individualite" ${ }^{26}$, de "la libre individualite" 27 .

Les dieux sont anthropomorphiques.

Il faut éviter ici une grave erreur d'interprétation. Hegel en ce

${ }^{21}$ Esthetique, II, p.149.

${ }^{22}$ Ansichten..., I, p. 196.

${ }^{23}$ Esthetique, II, p.149.

${ }^{24}$ Ibid., p. 150.

${ }^{25}$ Ibid., I, p. 264.

${ }^{26}$ Forster, Phil. Schriften, p. 170; Hegel, Philosophie de l'histoire, trad. française de J. Gibelin, p. 216.

${ }^{27}$ Esthétique, II, p. 155. 
qui touche la religion grecque, ne songe pas du tout à une religion artificielle au sens où elle aurait été inventée, fabriqué de toute pièce par des gens qui ne croiraient pas en ses dieux, et qui se seraient rendu coupables, envers autrui, d'une sorte d'imposture. Il n'adopte pas la philosophie de l'Aufklärung, la philosophie des Lumières qui voit dans la religion en général, et aussi dans la religion grecque en particulier, un ensemble de fables composées à l'usage des peuples crédules par des prêtres trompeurs et des tyrans perfides. Encore qu'il n'exclue pas un usage perfide de la religion, il conçoit la religion grecque comme l'expression authentique et nécessaire des aspirations du peuple grec, de l'esprit grec: une idéologie. Les Grecs croyaient à leurs Dieux. Leur création des dieux était spontanée, fervente, et, en outre, belle. Il s'agit donc plus d'une religion artistique que d'une religion artificielle. L'artiste grec ne feint pas d'être religieux, il ne tient pas ses dieux pour factices. Mais, sans prendre parfaitement conscience de l'opération qu'il effectue, il sculpte des dieux auxquels il voue ensuite un culte. Il s'éprend, mais religieusement, de ses oeuvres.

Selon Hegel, la religion grecque, par son caractère artistique, oeuvre des hommes, se distingue, d'une part de la religion naturelle dans laquelle les objets d'adoration ne sont pas produits par l'homme mais découverts par lui dans la nature, d'autre part de la religion chrétienne, où l'être adoré n'est plus ni sensible ni extérieur à l'homme.

Nous rencontrons ici la préfiguration de ce que sera une explication anthropologique de la religion, telle que l'exposera plus tard Feuerbach. La divinité, créee par l'artiste, incarne ses sentiments et ses aspirations. Les hommes, par l'intermédiaire des artistes, projettent idéalement dans les dieux ce qu'ils trouvent en eux-mêmes de meilleur et de plus beau. Ensuite ils attribuent une existence indépendante à ces abstractions qu'ils dotent de tous les pouvoirs et qu'ils ressentent ensuite comme des puissances supérieures et parfois contraignantes. La postérité en réclamera la restitution, et Hegel l'approuve: "Ce fut un mérite réservé à notre temps que de revendiquer comme propriété de l'homme les trésors qui ont été dépensés aux cieux ${ }^{\text {"28. }}$.

Si l'on adopte, ne serait-ce que momentanément, cette vision de la naissance simultanee de la religion et de l'art grecs, alors on doit aussi envisager leur mort: "Tout ce qui est né, mérite de périr." Forster, Hegel et Marx sont dotés d'un grand sens historique, encore qu'à un degré inégal et d'une manière différente. Ils se représentent les choses

${ }^{28}$ Hegels Theologische Jugendschriften, p. 225. 
dans leur devenir. Donc, la Kunstreligion, ce culte de la belle forme humaine idéalisée, cette belle réussite, cette belle harmonie, ne pouvait durer qu'un moment. L'art grec a succombé. A des titres divers, le regret de cette perte s'exprime chez Forster, chez Hegel et chez Marx.

Selon Forster, les chefs-d'oeuvre grecs étaient crés dans l'enthousiasme que suscite l'amour de la patrie. ils magnifiaient le zèle passionné du citoyent pour la République, ils présentaient le beau socialement moral: das Sittlichschöne. Pour Hegel, ils exprimaient directement "l'ordre éthique", "l'esprit vrai"29.

Les deux philosophes voient Kunst et Sittlichkeit s'épanouir ensemble: "C'est d'un seul sentiment que jaillissent l'art et la vertu", dit Forster ${ }^{30}$, et pour lui il s'agit de la vertu du républicain, qui ne sépare pas son sort de celui de sa libre patrie, de la totalité éthique à laquelle il appartient.

Le Grec confondait sa vie privee avec la vie publique. Aussi l'art grec était-il un art public. Forster le remarquait:

\begin{abstract}
Jusqu'à l'époque de Périclès, alors qu'Athènes la fière, avec une légèreté juvénile, prodiguait les millions pour l'embellissement de la ville et la somptuosité des fêtes publiques, le luxe privé resta enfermé dans des limites étroites. Les habitations, le mobilier, les vêtements, les repas, tout révélait encore la modération et la simplicité des moeurs domestiques ${ }^{31}$.
\end{abstract}

Hegel notera également que ... "les Grecs n'utilisaient le faste et la beauté que dans leurs édifices publics; leurs habitations privées étaient insignifiantes ${ }^{\text {n32 }}$.

Mais cette situation changera complètement lorsque le despotisme aura chassé la liberté politique et que l'intérêt privé, le commerce, l'argent étendront leur emprise sur les esprits. Alors s'édifiera ce que Hegel appelle l'Etat du droit, sur les ruines de la Sittlichkeit.

Le sentiment unique d'où jaillissent ensemble l'art et la vertu, "le souffle froid du despotisme - nous dit Forster -, l'avait flétri. L'amour de la patrie ne pouvait plus exalter celui qui avait, non plus une patrie, mais un maître" ${ }^{\mathrm{m} 33}$.

Hegel retrouvera de tels accents pour décrire l'existence malheu-

\footnotetext{
${ }^{29}$ Phil. Schriften, p. 125.

${ }^{30}$ Ibid., p. 126.

${ }^{31}$ Ibid., p. 126.

${ }^{32}$ Esthetique, III, Ière partie, p. 80.

${ }^{33}$ Phil. Schriften, p. 127.
} 
reuse des personnes privées, courbées sous le joug d'un maître.

Quant aux effets de ces événements sur l'art, Hegel utilisera pour les illustrer le même exemple que Forster, celui de Lucullus. Aux statues des dieux, crées pour le peuple et publiquement proposées à son adoration, Forster avait en effet opposé "les oeuvres d'art auxquelles un Lucullus égoïste laisse rendre hommage dans ses palais" ${ }^{\text {"34 }}$.

$A$ une ère de liberté, pendant laquelie les artistes avaient exprimé les impressions naïves du peuple, va succéder une ère de tyrannie, de cupidite, de calcul sordide, de ratiocination desséchante. L'art en mourra.

On ne peut méconnaître dans ces considérations un pressentiment, mais un pressentiment seulement, des idées que Marx, partant d'autres prémisses philosophiques, sera en mesure de développer beaucoup plus précisément et adéquatement.

L'égoïsme, le luxe privé - et donc la domination de plus en plus grande de la propriété privée, l'affaiblissement du sentiment social et patriotique, provoqueront la mort de la "religion de l'art" et favoriseront l'apparition d'une autre religion, la religion "privée" par excellence, le christianisme: voilà ce que suggèrent Forster et Hegel.

Marx rencherit sur ce thème. Mais, dans le texte concerné, c'est curieusement sous forme interrogative qu'il évoque l'incompatibilité de certaines productions artistiques avec le développement du capitalisme moderne, dont il envisage ici surtout l'aspect technique:

La façon de voir la nature et les rapports sociaux qui inspire l'imagination grecque et constitue de ce fait le fondement de la mythologie grecque est-elle compatible avec les chemins de fer, les locomotives et le télégraphe électrique ${ }^{35}$ ?

La réponse implicite est évidente: non! Pour que l'art grec fût ce qu'il a été, il fallait une mythologie grecque qui supposait elle-même un développement technique rudimentaire.

Marx en arrive même à formuler une hypothèse inquiétante:

Il faut en tout cas une mythologie. Donc en aucun cas une societé arrivé à un stade de développement excluant tout rapport mythologique avec la nature, tout rapport generateur de mythes, exigeant donc de l'artiste une imagination indépendante de la mythologie ${ }^{36}$.

\footnotetext{
${ }^{34}$ Hegel, Werke (éd. Lasson), t. XX, p. 266.

${ }^{35}$ Marx, Op. cit., p. 174.

${ }^{36}$ Ibid., p. 174.
} 
Alors pas d'art sans mythologie?

Le progrès technique efface les conditions de création des formes les plus "grecques" de l'art, et, par exemple, de l'épopée, dont Marx admire le modèle dans l'oeuvre d'Homère.

Hegel a estimé que l'art, dans l'extinction de toute mythologie, perdait son sens, sinon son existence. Les oeuvres d'art grecques ne gardent un attrait pour nous que parce que nous ne les comprenons plus du tout à la manière des Grecs. Cette désaffection vaut d'ailleurs aussi bien pour la peinture ou la sculpture chrétienne. Dans les statues, nous ne voyons plus les dieux eux-mêmes:

\begin{abstract}
Nous avons beau trouver les images des dieux grecs incomparables, et quelles que soient la dignité et la perfection avec laquelle sont représentés Dieu le Père, le Christ, la Sainte Vierge, quelle que soit l'admiration que nous éprouvons à la vue de ces statues, cela n'y fait rien: nous ne plions plus les genoux ${ }^{37}$.
\end{abstract}

Le Beau idéal, qui est en même temps le beau socialement moral, ne peut garder sur les modernes l'empire qu'il exerçait sur les Grecs. Car, nous dit Forster,

cela ne serait possible que dans un cas: si l'esprit de l'époque (der Geist des Zeitalters) n'avait pas eu d'effet sur l'artiste; si, indépendamment du temps et des circonstances, le génie artistique, planant dans une perfection abstraite, était resté un Grec au milieu des chrétiens ${ }^{38}$.

Or il n'en est rien. L'artiste n'est pas "indépendant du temps et des circonsctances." Au contraire, il est leur porte-parole, et Hegel le précisera: "Tout individu est fils de son temps" 39 .

Dans ces conditions, nous confie Forster, "les figures grecques et les dieux grecs (...) nous sont devenus aussi étrangers que des sons et des noms prononcés en grec dans notre poésie ${ }^{140}$.

Dans une des plus belles pages de la Phénoménologie, Hegel décrit à son tour ce destin de la Kunstreligion:

les statues sont maintenant des cadavres dont l'âme animatrice s'est enfuie, les hymnes sont des mots que la foi a quittes (...). Ces oeuvres

${ }^{37}$ Esthetique, I, p. 137.

${ }^{38}$ Ansichten, p. 206.

${ }^{39}$ Histoire de la philosophie, Introduction, trad. Gibelin, p. 135.

${ }^{4}$ Ansichten..., p. 207. 
sont désormais ce qu'elles sont pour nous: de beaux fruits détachés de l'arbre (...). Notre opération, quand nous jouissons de ces oeuvres (...) est l'operation exterieure qui purifie ces fruits de quelques gouttes de pluie ou de quelques grains de poussière, et à la place des éléments intérieurs de l'effectivité éthique qui les environnait, les engendrait et leur donnait l'esprit, établit l'armature interminable des éléments morts de leur existence extérieure, le langage, l'élément de l'histoire, etc., non pas pour pénétrer leur vie, mais seulement pour se les représenter en soi-même ${ }^{41}$.

Les oeuvres perdent leur signification religieuse quand disparaît la base spirituelle sur laquelle elles se fondaient. Ce qui, après transposition, se comprend assez bien aussi dans la perspective de Marx: l'art change avec ses conditions sociales. La base entraîne dans sa ruine la "superstructure" qu'elle portait.

Voici la conclusion commune aux trois theoriciens: la disparition de l'art grec, en tant qu'activité créatrice, est irréversible. Sa base spirituelle, ses conditions matérielles et sociales sont définitivement périmées. Marx ne prononce qu'une rapide oraison funebre: "les conditions sociales insuffisamment mûres où cet art né, et où seulement il pouvait naître, ne pourront jamais revenir ${ }^{n 42}$.

C'est précisément cette notion de maturité insuffisante qui lui suggère une réponse possible à la question qui le tourmente. Ni Forster ni Hegel ne peuvent imaginer une solution valable, parce qu'ils ne rapportent pas objectivement et convenablement le progrès de l'art au progrès de la vie économique, technique et sociale. Ils ne sont pas en mesure de rattacher un type de succès artistique à une forme de retard technique. Or c'est justement cette sorte de décalage qui fournira à Marx un élément d'explication. En ce qui concerne l'activité pratique et la compréhension des structures sociales, les Grecs étaient par rapport a nous des naïfs, moins aliénés que nous dans une société moins déchirée que la nôtre, du moins en ce qui concernait les hommes libres de la Cite. On peut donc comparer légitimement leur situation esthétique par rapport à celle du monde moderne à la situation de l'enfant par rapport à l'adulte.

L'adulte ne redevient pas un enfant, mais il réintériorise ce que l'enfant avait extériorisé, exprimé, et dans cette mesure lui donne une nouvelle vie. Marx propose de cette récupération esthétique de l'enfance dans la maturité de fort belles images, mais il ne le fait prudemment, là-

\footnotetext{
${ }^{41}$ Phenomenologie, II, p. 261.

${ }^{42}$ Marx, Op. cit., p. 175.
} 
aussi, que sur le mode interrogatif:

L'homme ne prend-il pas plaisir à la naïveté de l'enfant et, ayant
accédé à un niveau supérieur, ne doit-il pas aspirer lui-mème à
reproduire sa vérité? Dans la nature enfantine, chaque epoque ne
voit-elle pas revivre son propre caractere dans sa vérité naturelle?
Pourquoi l'enfance historique de l'humanité, là où elle a atteint son
plus bel épanouissement, pourquoi de stade de développement révolu
à jamais n'exercerait-il pas un charme éternel? ${ }^{43}$

Pourquoi pas, en effet? Mais pourquoi pas, aussi bien, le contraire? Pourquoi nos contemporains ne mépriseraient-ils pas les statues grecques en même temps que leur caractère religieux: pourquoi ne les oublieraient-ils pas, ou ne se désintéresseraient-ils pas de tout ce passé architectural, sculptural ou poétique comme ils se détournent du mode d'activité technique qui l'accompagnait chronologiquement?

On pourrait répondre plus décidément à ces questions si l'on élargissait maintenant le champ de leur application.

Il conviendrait d'examiner le sens nouveau qu'elles prennent après l'apparition et la disparition de tant de styles et d'écoles artistiques divers, depuis Marx. L'activité créatrice s'est orientée dans de tout autres directions. Pour ne s'arrêter qu'à un seul exemple, doit-on après l'extraordinaire floraison de l'impressionnisme, en peinture, maintenir que les Grecs ont fourni au genre humain des "modeles inaccessibles"? Ni Monet ni Renoir n'ont tenu le passé pour intouchable. Mais cela n'implique aucun mépris, seulement un éloignement. Picasso lui-même s'est formé dans des académies de peinture où l'on dessinait d'après des modèles grecs. Quand, plus tard, il cultive audacieusement son originalite personnelle, il leur doit encore beaucoup, même dans le rejet apparent et la contradiction. Sans vergogne, il va chercher des modeles dans les vieilles civilisations africaines ou sud-américaines.

La relation du présent et du passé, en art, se révèle très subtile. Mais, à la limite, elle ne respecte aucun tabou.

Pour intervenir plus universellement et valablement dans cette problématique, il faudrait considérer aussi d'autres cultures que celles de l'Europe. Marx, à cause des limites de sa formation et de son experience, pourtant vastes, ne pouvait faire remonter le développement significatif de l'art qu'à la Grece antique, en la préférant à d'autres cultures qu'il connaissait. Mais celles-ci lui étaient beaucoup moins familières et,

${ }^{43}$ Ibid., p. 175. 
surtout, beaucoup de trésors artistiques du monde lui restaient presque inconnus: par exemple, ceux de l'Asie, de la Chine.

Il considérait les Grecs comme les "enfants normaux" du genre humain. C'était là un européocentrisme inévitable en son temps, mais que les événements mondiaux ultérieurs ont permis de dépasser. L'art européen contemporain emprunte des modeles et une inspiration à tous les continents.

Les hommes de notre temps recueillent un héritage esthétique de plus en plus riche, en quantité et en diversité. L'art grec antique y conserve son prestique, mais perd peu à peu de son exclusivite, pour faire sereinement place aussi aux autres, afin d'encourager toujours mieux l'élan universel de création et de progrès. Reste que l'on peut utilement se demander si les profondes considérations de Marx à son propos valent aussi pour toutes les autres formes de cuiture. 